\title{
Imaging of adenosine bolus transit following intravenous administration: insights into antiarrhythmic efficacy
}

\author{
G A Ng, W Martin, A C Rankin
}

\begin{abstract}
Objective-To study the effects of the site of intravenous injection of adenosine and to assess the site of action of adenosine in the heart by correlating cardiac effects with bolus transit.

Methods-Ten patients undergoing routine technetium (Tc-99m) gated blood pool ventriculography consented to the coadministration of intravenous adenosine. The dose of adenosine required to produce heart block during sinus rhythm was determined following antecubital vein administration. This dose $(6-18 \mathrm{mg})$ was mixed with $\mathrm{Tc}-99 \mathrm{~m}$ and given first into the same antecubital vein (proximal injection) and then repeated into a hand vein (distal injection). The ECG was recorded and the transit of the bolus was imaged using a gamma camera.

Results-Heart block occurred in all 10 patients (second degree in seven, first degree in three) at (mean (SEM)) 17.5 (1.0) seconds after the proximal injection of adenosine. Distal injection produced heart block in six patients (second degree in two, first degree in four) at 21.9 (4.4) seconds $(p<0.01)$. In eight of 10 patients the electrophysiological effects were less with distal injection. The onset of heart block was close to the time of peak bolus Tc-99m activity in the left ventricle. Peak bolus activity was delayed (by about three seconds) and the duration of bolus activity in the left ventricle was increased with distal injection compared with proximal injection, at $17.2(4.2) v 9.2$ (3.1) seconds, $\mathrm{p}<0.01$.
\end{abstract}

Conclusions-The lesser electrophysiological effects of adenosine following distal intravenous injections were associated with delay in transit time and dispersion of the bolus. The correlation of adenosine induced heart block with bolus activity in the left heart indicated dependence on coronary arterial delivery of adenosine to the atrioventricular node.

(Heart 1999;82:163-169)

Keywords: adenosine; antiarrhythmics; nuclear medicine; imaging

Adenosine is an endogenous purine nucleoside which, when injected intravenously, causes transient slowing of the heart rate, in part from sinus node slowing but more importantly from atrioventricular (AV) nodal block. ${ }^{12}$ The negative dromotropic action has made it of value in the diagnosis and treatment of tachyarrhythmias in the acute setting. ${ }^{3}{ }^{4}$ The plasma half life of adenosine is short (less than two seconds) ${ }^{5}$ and its cardiac actions are correspondingly brief, which is an advantage because any adverse effects will also be of short duration. However, it may also be disadvantageous because its efficacy is dependent on the site and method of intravenous injection. ${ }^{6}$ Substantial removal and metabolism of adenosine may occur during transit from a peripheral vein to the heart. We therefore studied the effect of adenosine administration at two "peripheral" venous sites on the electrophysiological actions and bolus transit characteristics.

The negative dromotropic action of adenosine has been shown experimentally during superfusion of isolated right heart preparations. ${ }^{7}$ It is not known whether adenosine acts on the sinus and AV nodes in humans by direct contact in the right atrium or by perfusion of the nodal tissues through the coronary arteries following passage through the pulmonary vasculature and the left heart. We used a nuclear imaging technique to correlate the transit of the bolus with the timing of the electrophysiological actions of adenosine. 18 March 1999

Table 1 Details of the patients

\begin{tabular}{|c|c|c|c|c|c|c|c|}
\hline Patient & Age (years) & Diseases & Cardiac drug treatment & Study dose (mg) & $\begin{array}{l}\text { Resting heart rate } \\
\text { (beats/min) }\end{array}$ & $\begin{array}{l}\text { Body weight } \\
\text { (kg) }\end{array}$ & Response \\
\hline 1 & 43 & MI & Nitrate, aspirin & 6 & 88 & 86 & $\mathrm{SS}, 1^{\circ} \mathrm{AVB}$ \\
\hline 2 & 59 & - & Atenolol, nitrate & 12 & 63 & 76 & $\mathrm{SS}, 1^{\circ} \mathrm{AVB}, 2^{\circ} \mathrm{AVB}$ \\
\hline 3 & 51 & $\begin{array}{l}\text { Hypertension, } \\
\text { NIDDM }\end{array}$ & Atenolol, diltiazem, nitrate, aspirin & 12 & 63 & 100 & $\mathrm{SS}, 1^{\circ} \mathrm{AVB}, 2^{\circ} \mathrm{AVB}$ \\
\hline 4 & 51 & - & Aspirin & 18 & 90 & 85 & $\mathrm{SS}, 1^{\circ} \mathrm{AVB}, 2^{\circ} \mathrm{AVB}$ \\
\hline 5 & 40 & - & Atenolol, aspirin & 18 & 56 & 70 & $\mathrm{SS}, 1^{\circ} \mathrm{AVB}, 2^{\circ} \mathrm{AVB}$ \\
\hline 6 & 47 & CVA & Nifedipine, aspirin & 6 & 60 & 70 & $\mathrm{SS}, 1^{\circ} \mathrm{AVB}, 2^{\circ} \mathrm{AVB}$ \\
\hline 7 & 28 & MI & Aspirin & 12 & 66 & 108 & $\mathrm{SS}, 1^{\circ} \mathrm{AVB}, 2^{\circ} \mathrm{AVB}$ \\
\hline 8 & 64 & MI, CABG & Atenolol, diltiazem, nitrate, aspirin & 12 & 60 & 62 & $\mathrm{SS}, 1^{\circ} \mathrm{AVB}, 2^{\circ} \mathrm{AVB}$ \\
\hline 9 & 39 & MI & Atenolol, diltiazem, nitrate, aspirin & 12 & 60 & 70 & $\mathrm{SS}, 1^{\circ} \mathrm{AVB}, 2^{\circ} \mathrm{AVB}$ \\
\hline 10 & 57 & - & Atenolol, diltiazem, nitrate, aspirin & 12 & 60 & 70 & $\mathrm{SS}, 1^{\circ} \mathrm{AVB}, 2^{\circ} \mathrm{AVB}$ \\
\hline
\end{tabular}

CABG, coronary artery bypass graft; CVA, cerebrovascular accident; MI, myocardial infarction; NIDDM, non-insulin dependent diabetes mellitus; SS, sinus slowing; $1^{\circ} \mathrm{AVB}$, first degree atrioventricular block; $2^{\circ} \mathrm{AVB}$, second degree atrioventricular block. 

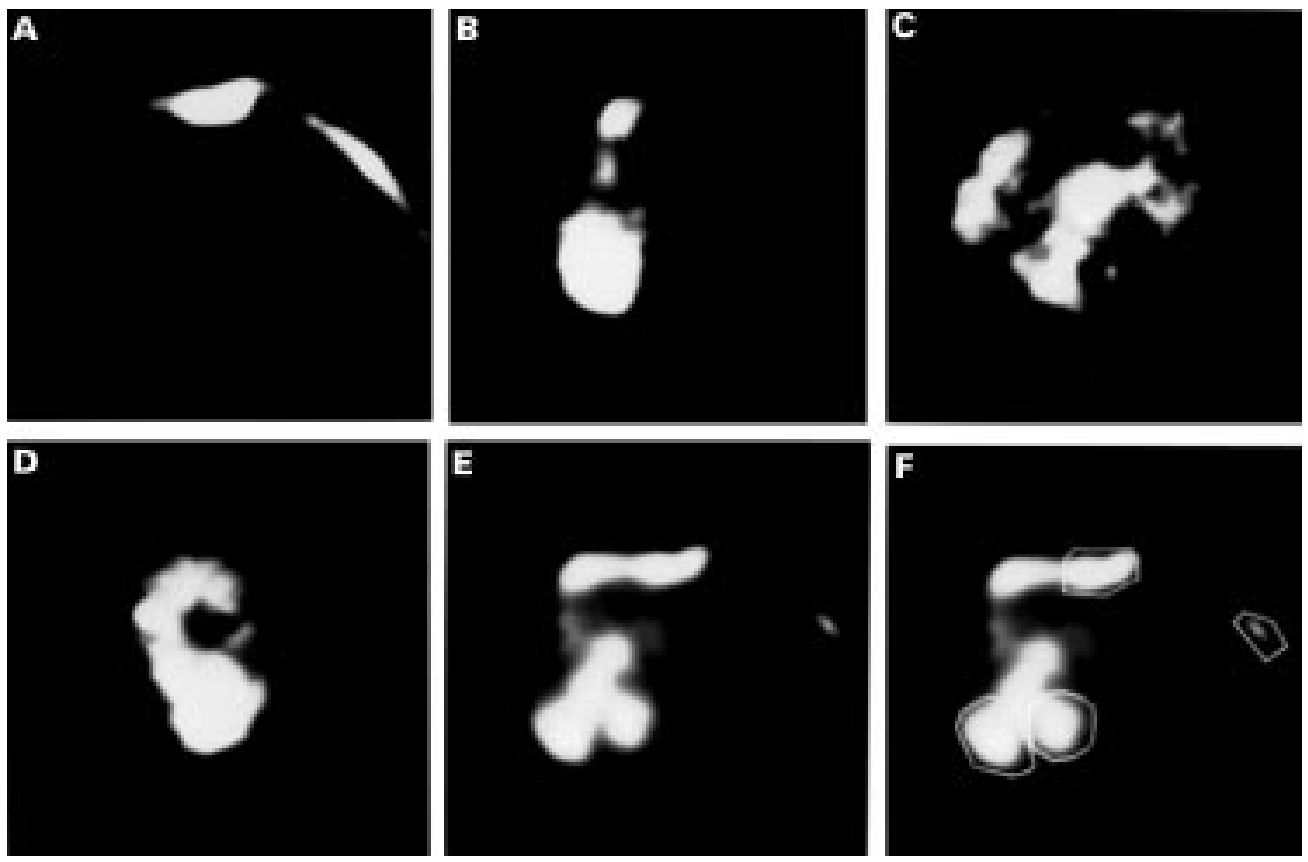

Figure 1 Activity of adenosine/technetium-99m bolus imaged during transit from (A) left upper arm and shoulder through (B) right ventricle, $(C)$ pulmonary artery and lungs to (D) left ventricle and aorta. The totalised image from frames captured during the transit is shown in (E) and the four regions of interest-left upper arm, left shoulder, right ventricle, and left ventricle - are shown (F).
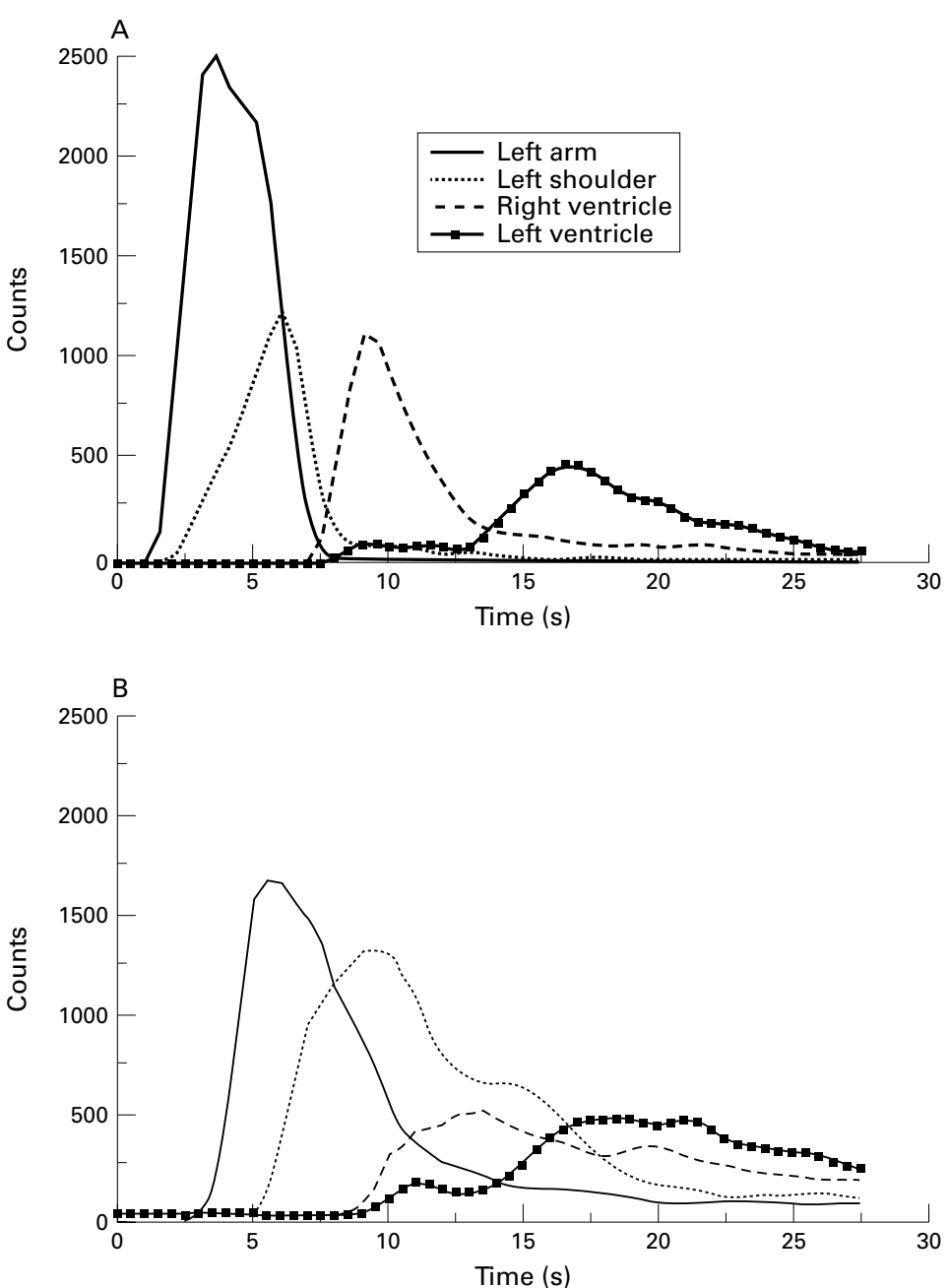

Figure 2 Activity-time curves at the four regions of interest following $(A)$ the proximal injection and (B) the distal injection.
The aims of this study were to assess the effects of the site of intravenous injection of adenosine and to determine the site of action of adenosine in the heart.

\section{Methods}

Patients undergoing technetium (Tc-99m) gated blood pool ventriculography for clinical indications were studied. The study was approved by the medical ethics committee of Glasgow Royal Infirmary. Written informed consent was obtained from each patient, who was warned of the systemic effects of adenosine injections including chest discomfort, flushing, and breathlessness. Patients with a history of asthma or symptoms suggestive of unstable angina or congestive heart failure were excluded. Patients taking dipyridamole (an adenosine uptake blocker) or theophylline (a competitive antagonist of adenosine) were also excluded.

Ten male patients were studied aged 28 to 64 years, with a mean age of 48 years. Their characteristics are shown in table 1. All had a history of chest pain as the clinical indication for investigation with exercise thallium scanning and blood pool ventriculography. The stress thallium scans were abnormal in all of the patients and subsequent coronary angiography confirmed the presence of coronary artery disease. Eight of the patients were taking antianginal treatment (table 1). All patients were in sinus rhythm.

TEST ADMINISTRATION OF ADENOSINE

Adenosine (3 mg/ml; Adenocor, Sanofi Winthrop, Guildford, UK) was given as a rapid bolus through an 18 gauge cannula in a large left antecubital fossa vein, followed by a $10 \mathrm{ml}$ saline flush, to mimic its administration in the 
Proximal

Distal

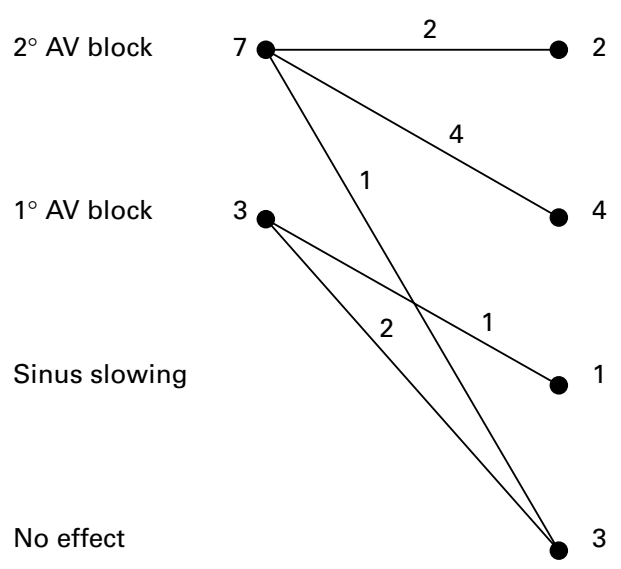

Figure 3 ECG responses produced by the proximal and distal injections of adenosine.

management of tachyarrhythmias. Incremental doses of adenosine were given during sinus rhythm and the electrophysiological effects were determined from a continuous ECG recording at $25 \mathrm{~mm} / \mathrm{s}$. First degree AV block was defined by a prolongation of PR interval to more than 0.2 second and second degree block was defined by the absence of QRS complex after a $P$ wave. Increasing doses of adenosine, by $3-6 \mathrm{mg}$ increments, were given up to a maximum of $18 \mathrm{mg}$ until second degree AV block occurred or side effects limited further increments. The minimum dose of adenosine required to produce $\mathrm{AV}$ block was used as the study dose in the subsequent nuclear imaging with Tc-99m.

IMAGING AND ECG DATA ACQUISITION DURING ADENOSINE BOLUS INJECTIONS

Gated blood pool radionuclide ventriculography was performed after an exercise thallium perfusion scan. All patients had undergone a symptom limited bicycle ergometric exercise test with a subsequent 20 minute supine rest period for thallium perfusion imaging, followed immediately by technetium gated blood pool imaging. In routine clinical practice, $600 \mathrm{MBq}$ of Tc-99m in a $3 \mathrm{ml}$ bolus would be given intravenously, following pretreatment with stannous pyrophosphate to ensure binding of the Tc-99m to red blood cells. In our study, the Tc-99m dose was divided to allow two separate injections to be imaged. For the first injection, the previously determined study dose of adenosine was mixed with $200 \mathrm{MBq}(1 \mathrm{ml})$ of Tc-99m. The second injection contained 400 $\mathrm{MBq}(2 \mathrm{ml})$ of Tc-99m and the same dose of adenosine. Both injections were made up to the same volume by the addition of saline. A more concentrated adenosine solution $(5 \mathrm{mg} / \mathrm{ml})$, prepared by the pharmacy department in our hospital, was used to keep the bolus volume close to that of the test injection.

The first injection (proximal injection) was given through the left antecubital cannula, followed by a $10 \mathrm{ml}$ saline flush. The ECG was monitored continuously. The beginning and the end of the bolus injection, the end of the saline flush, and the timing of the onset of the patient's symptoms were noted by marking on the continuously running ECG recording. Patients were asked to comment on the severity of the symptoms experienced with the injections, on a scale from 0 to 10 . Imaging was performed using a GE 300A mobile gamma camera with a high sensitivity $30 \mathrm{~cm}$ parallel collimator at a $10^{\circ}$ left anterior oblique projection and data acquisition was continued for two minutes, with $64 \times 64$ resolution images acquired at one second intervals. The beginnings of the ECG recording and the nuclear imaging were synchronised so that the temporal events observed could be directly correlated.

When the heart rate had returned to control values, which took approximately five minutes, the second injection (distal injection) was given through an 18 gauge cannula inserted into a
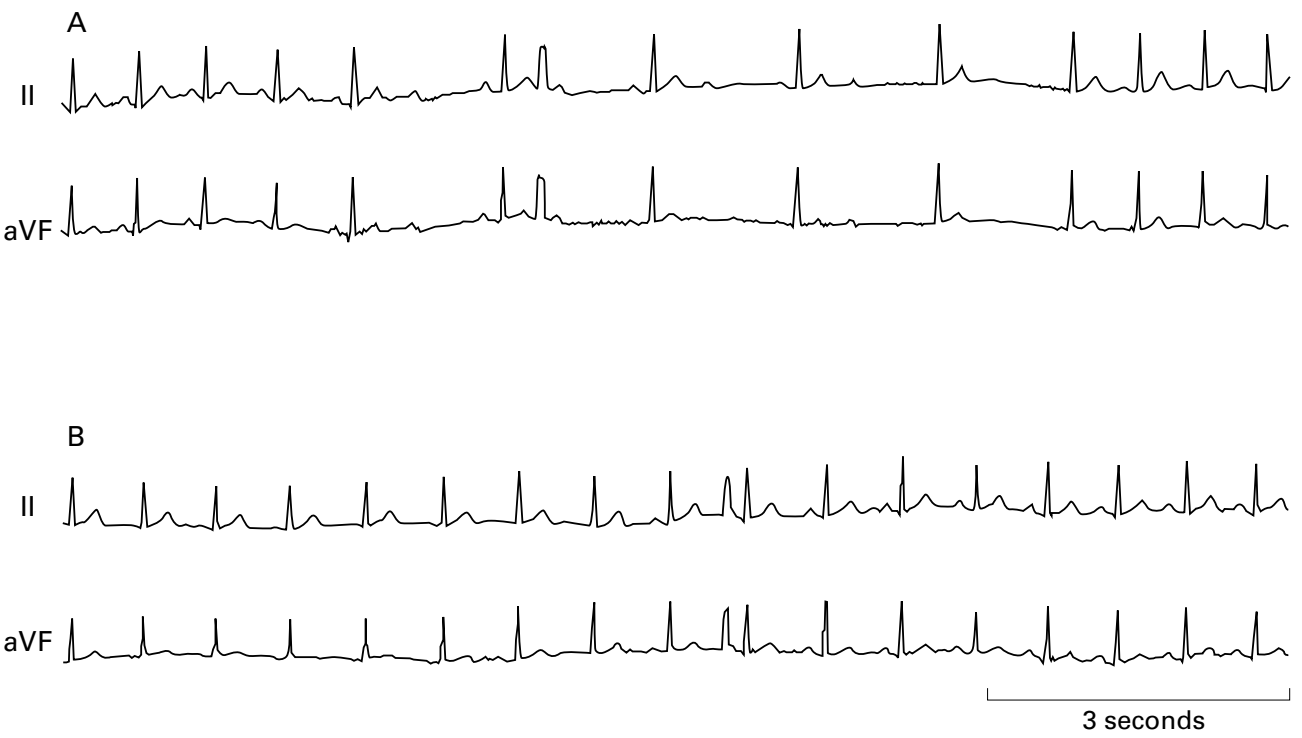

Figure 4 ECG recordings following (A) proximal injection of adenosine, showing second degree AV block, and (B) distal injection of adenosine in the same patient, which did not produce AV block. Adenosine was given 16 seconds before beginning these recordings. Marker deflections on the tracings correspond to the patient's reporting of systemic symptoms. (Surface ECG leads II and aVF, $25 \mathrm{~mm} / \mathrm{s}$.) 
Table 2 Adenosine bolus transit: the timing of bolus arrival, peak bolus activity, and bolus duration at the four regions of interest (ROI) following the proximal and distal adenosine injections

\begin{tabular}{lllll}
\hline ROI & Bolus & Proximal injection $(s)$ & Distal injection $(s)$ & p Value \\
\hline Left upper arm & Arrival & $0.8(0.2)$ & $3.1(0.6)$ & $<0.001$ \\
& Peak & $1.4(0.2)$ & $4.5(0.6)$ & $<0.001$ \\
& Duration & $4.3(0.3)$ & $7.4(1.4)$ & $<0.05$ \\
Left shoulder & Arrival & $2.3(0.5)$ & $4.7(0.6)$ & $<0.01$ \\
& Peak & $3.6(0.6)$ & $6.1(0.8)$ & $<0.02$ \\
& Duration & $4.4(0.4)$ & $5.8(0.5)$ & $<0.05$ \\
Right ventricle & Arrival & $6.8(0.7)$ & $8.8(0.7)$ & $<0.05$ \\
& Peak & $8.2(0.7)$ & $11.1(0.8)$ & $<0.01$ \\
& Duration & $4.6(0.6)$ & $11.9(1.4)$ & $<0.001$ \\
Left ventricle & Arrival & $12.6(0.8)$ & $13.6(0.8)$ & 0.1 \\
& Peak & $15.8(0.7)$ & $19.3(1.1)$ & $<0.005$ \\
& Duration & $9.2(1.0)$ & $17.2(1.3)$ & $<0.001$ \\
\hline
\end{tabular}

Values are mean (SEM). frame in each region was calculated. Activitytime curves at each region were then constructed (fig 2). The time of arrival of the bolus and the time to peak bolus activity were obtained from the activity-time curves. Bolus duration was taken as the time interval between the bolus activity reaching half maximum and decreasing to half maximum. The time of arrival of the bolus, time to peak bolus activity, and bolus duration at each region of interest were correlated with the timing of the ECG changes.

STATISTICS

Values are presented as mean (SEM). The difference between the proximal and distal injections in producing heart block was analysed using the $\chi^{2}$ test. A paired two tailed Student's $t$ test was used for comparing other effects of the proximal and distal injections. A probability $(p)$ value of less than 0.05 was considered significant. the ECG recording, event marking, and imaging were repeated as for the first injection.

ANALYSIS

The times for the beginning and the end of the injection boluses, the end of the saline flush, the onset of electrophysiological effects (including sinus slowing and first and second degree AV block), and the onset of systemic symptoms were measured from the ECG tracings for the two injections and compared.

Data acquired by the gamma camera were analysed using a computer. The activity of the adenosine/Tc-99m bolus was imaged during transit from the arm to, and through, the heart (fig 1). On the computer display of stored data, four regions of interest were drawn around the activity from the left upper arm, left shoulder, right ventricle, and left ventricle. The period of data acquisition was analysed in half second frames and the magnitude of bolus activity per

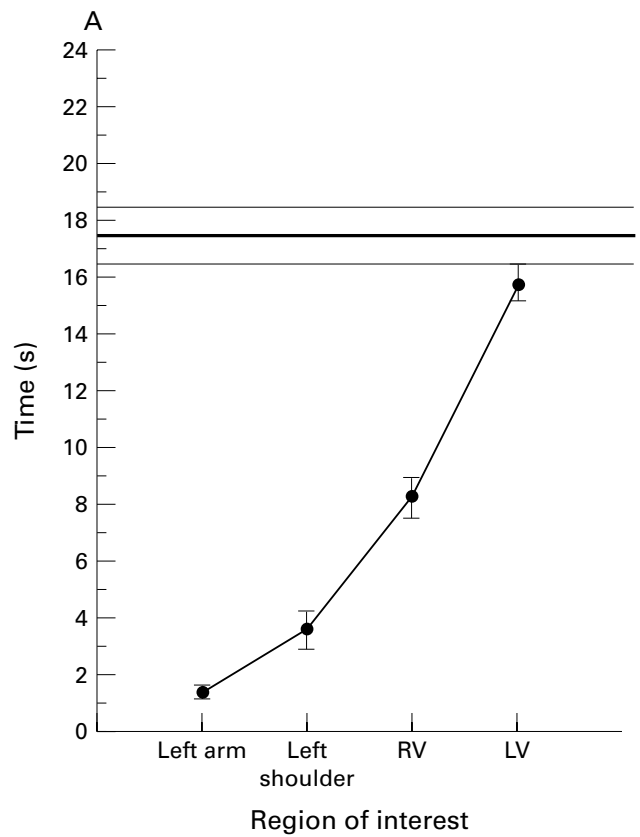

\section{Results}

TEST DOSAGES OF ADENOSINE

The test injection of adenosine (6-18 mg) caused second degree AV block in nine of the patients (table 1) - one with $6 \mathrm{mg}$, six with 12 $\mathrm{mg}$, and two with $18 \mathrm{mg}$. These doses were used for the imaging study. One patient had sinus slowing and first degree AV block, but not second degree AV block, in response to 6,12 , and $18 \mathrm{mg}$ of adenosine; $6 \mathrm{mg}$ was used as the study dose for this patient. The mean (SEM) heart rate before administration of the test doses was 65 (3) beats/min. There was no correlation between the test dose and the body weight of the patient or the heart rate before the injection.

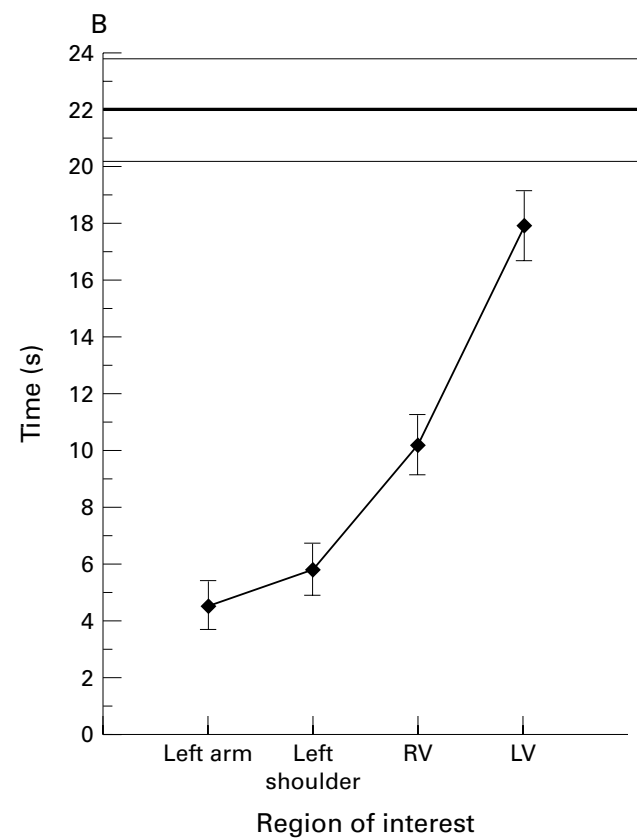

Figure 5 Time to peak bolus activity (mean and standard error bars) at each of the four regions of interest for patients who had $A V$ block with $(A)$ the proximal injection, $n=10$, and $(B)$ the distal injection, $n=6$. The mean time of AV block following each injection is indicated by the thick horizontal lines and standard errors are shown as the thin lines. 

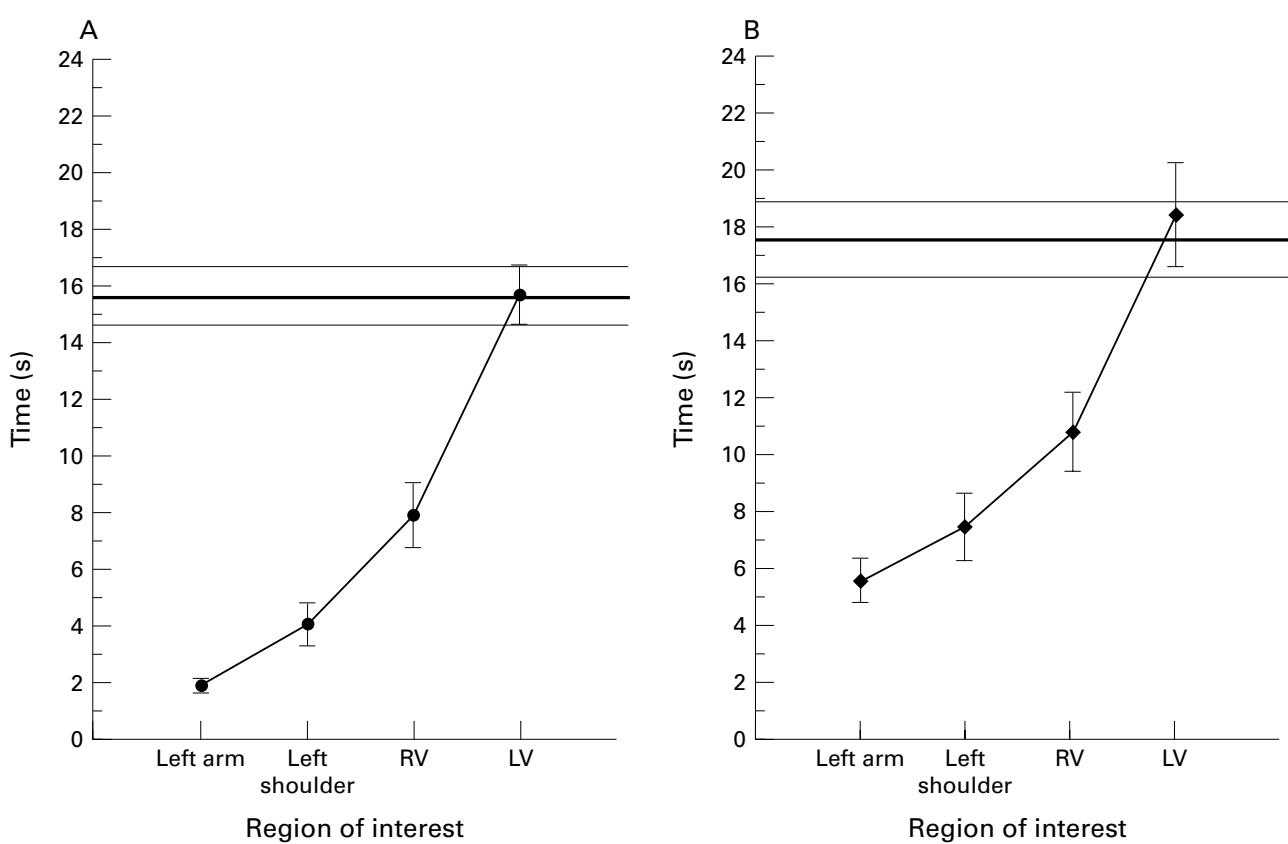

Figure 6 Time to peak bolus activity (mean and standard error bars) at each of the four regions of interest for patients who had sinus slowing with $(A)$ the proximal injection, $n=6$, and $(B)$ the distal injection, $n=5$. The mean time of sinus slowing following each injection is indicated by the thick horizontal lines and standard errors are shown as the thin lines.

ELECTROCARDIOGRAPHIC RESPONSES TO STUDY DOSES OF ADENOSINE

The mean heart rate at rest before the proximal injection ( 66 beats/min, range 49 to 95 ) was not different from that before the distal injection ( 66 beats/min, range 53 to 88 ; NS). There was no difference between the PR intervals before the proximal and distal injections (both 0.17 (0.01) seconds). The proximal injection was given within $0.9(0.2)$ seconds and the distal injection within $1.0(0.1)$ seconds (NS). The $10 \mathrm{ml}$ saline flush was completed slightly earlier in the distal injection (5.2 (0.2) seconds after the start of the injection) than in the proximal injection $(6.2(0.3)$ seconds after the start of the injection; $\mathrm{p}<0.05)$.

All 10 patients had heart block produced by the proximal injection of adenosine, with second degree AV block in seven patients and first degree block in three. By contrast, the distal injection produced second degree AV block in only two patients and first degree block in four $(p<0.05)$. In eight of the 10 patients, the ECG response to adenosine was less with the distal than with the proximal injection (fig 3). Figure 4 shows the ECG tracings for a patient who had second degree AV block with the proximal injection and no effect with the distal injection. First degree AV block preceded second degree block in seven patients with the proximal injection and in two patients with the distal. The time of onset of block was taken as that of the first degree AV block. Following the proximal injections in the 10 patients, mean time of onset of AV block occurred at 17.5 (1.0) seconds after the injection. With the distal injections, it occurred at 21.9 (1.9) seconds in the six patients who had AV blocksignificantly later than with the proximal injection in these patients (16.9 (1.5) seconds, $\mathrm{p}<0.05)$.
Adenosine caused slowing of the sinus rate with the proximal injection in six patients and in five of these with the distal injection. Sinus slowing preceded AV block when present. For the five patients who had sinus slowing with both injections, the percentage decrease in heart rate was greater and occurred earlier with the proximal injections than with the distal injections: $-20.8(3.8) \%$ at 14.4 (1.1) seconds $v-9.0(3.0) \%$ at $17.6(1.3)$ seconds, $\mathrm{p}<0.05$.

Patients experienced the systemic side effects of adenosine slightly earlier with the proximal than with the distal injections, at 19.1 $(0.9) v 21.3$ (1.0) seconds, $\mathrm{p}<0.05$. The symptoms were subjectively more severe with the proximal than with the distal injections: 7.3 $(0.7) \approx 6.0(0.6)$ on a scale from 1 to 10 ; $\mathrm{p}<0.05$.

TECHNETIUM/ADENOSINE BOLUS ACTIVITY

The timing of bolus arrival, peak activity, and the bolus duration at the four regions of interest with the proximal and distal injections are illustrated in fig 2 and summarised in table 2. The boluses arrived at the left upper arm, the left shoulder, and the right ventricle later with the distal than with the proximal injections, but this difference was not significant in the left ventricle. Peak bolus activity occurred significantly later with the distal than with the proximal injections in all four regions, with a mean delay of approximately three seconds at each region. The bolus durations were longer with the distal injections than with the proximal injections, this being progressively more significant along the route of transit of the boluses.

CORRELATION OF ELECTROPHYSIOLOGICAL EFFECTS AND ADENOSINE BOLUS TRANSIT The onset of electrophysiological effects of adenosine causing sinus slowing and AV block did not occur during passage through the right 

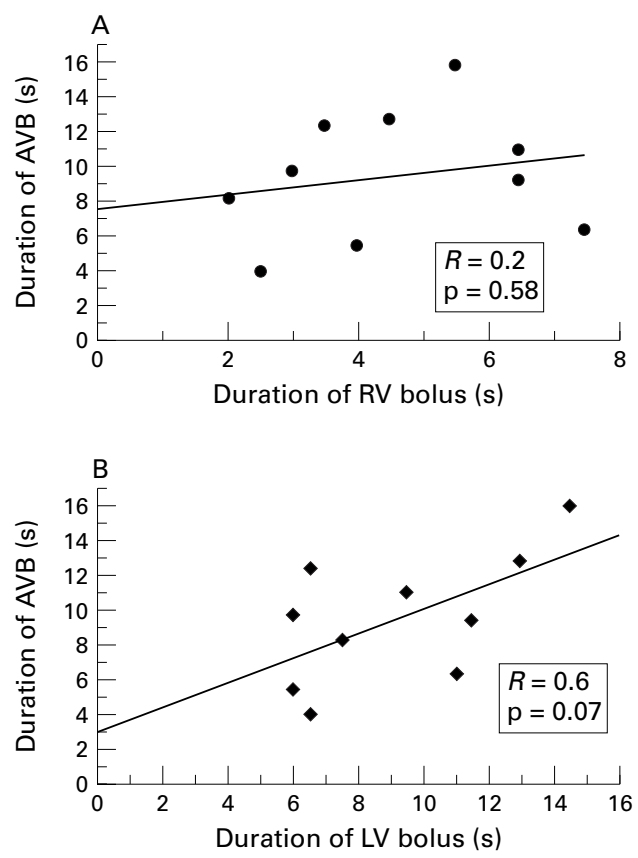

Figure 7 Correlation between the duration of AV block produced by adenosine injection (proximal) and the duration of bolus activity in $(A)$ the right ventricle and (B) the left ventricle.

heart but occurred close to the time of peak bolus activity in the left ventricle. Figure 5 shows the mean times of peak activity in the four regions of interest in relation to the time of onset of AV block. With the proximal injection, heart block occurred 9.5 (0.9) seconds after peak bolus activity in the right ventricle and only $1.7(0.6)$ seconds after peak bolus activity in the left ventricle. For the six patients who had AV block with the distal injection, heart block occurred 11.8 (1.0) seconds after peak bolus activity in the right ventricle but only 4.0 (1.3) seconds after peak bolus activity in the left ventricle. Similarly, sinus slowing correlated with passage through the left rather than the right ventricle (fig 6), although occurring earlier than heart block.

The relation between the duration of $\mathrm{AV}$ block following the proximal injections and the bolus duration in both ventricles is shown in fig 7. There was a closer correlation between the bolus duration in the left ventricle and the duration of $\mathrm{AV}$ block $(R=0.6, \mathrm{p}=0.07)$ than between the bolus duration in the right ventricle and the AV block duration $(R=0.2$, $\mathrm{p}=0.58)$.

\section{Discussion}

Adenosine induced AV block underlies the antiarrhythmic efficacy of intravenous adenosine in the treatment of paroxysmal supraventricular tachycardia, ${ }^{2-4} 6$ and its use during sinus rhythm for the diagnosis of accessory pathways $^{89}$ or for the assessment of catheter ablation procedures. ${ }^{10} 11$ The brevity of the electrophysiological effects of adenosine, because of its short plasma half life, ${ }^{5}$ has the benefit that adverse effects are also of short duration but the adverse effect that arrhythmias may recur immediately. ${ }^{6}$ The short half life has the further disadvantage of making the antiarrhythmic efficacy dependent on the route and speed of intravenous injection. Minimum effective doses of adenosine have been shown to be lower when given through central or femoral veins than through peripheral veins in patients with supraventricular tachycardia. ${ }^{6}{ }^{12}$ The present study shows marked differences in the electrophysiological effects of adenosine between two "peripheral" venous injection sites in the arm. The imaging of bolus transit provided insights into mechanisms underlying this importance of the route of administration.

Administration of the adenosine/Tc- $99 \mathrm{~m}$ bolus through the distal (hand) vein resulted in delay in peak activity (of approximately three seconds) and increased dispersion with prolongation of bolus duration. Both of these factors would contribute to the lesser effects of adenosine. Adenosine is rapidly removed from the circulation by cellular uptake and enzymatic metabolism. ${ }^{13}$ The half life of adenosine in human blood has been estimated to be 0.6 to 1.5 seconds, ${ }^{5}$ and thus even the short delay in transit is relevant. The increased dispersion of the bolus would result in dilution of the concentration of adenosine at its sites of action in addition to increasing contact with red blood cells and hence cellular uptake.

A threefold range in dose $(6-18 \mathrm{mg})$ required to produce second degree $\mathrm{AV}$ block during sinus rhythm was observed in the present study. Greater dosage variability, up to 10-fold (for example, $2.5-25 \mathrm{mg}$ ), has been reported in patients with supraventricular tachycardia. ${ }^{4}{ }^{6}$ Route of venous administration has been identified as one important variable ${ }^{6}$ but there is clearly additional individual variation in sensitivity to adenosine. The coadministration of other cardioactive drugs may be a factor. Verapamil and propranolol have been shown to have additive effects with adenosine in prolonging $\mathrm{AV}$ conduction time, although only verapamil reduced the dose of adenosine required to produce AV block. ${ }^{14}$ In the present study six patients were taking atenolol and four of these were also taking diltiazem (table 1). However, there was no evidence of enhancement of the negative dromotropic action of adenosine, as all the patients taking $\beta$ blockers and calcium antagonists requiring $12 \mathrm{mg}$ of adenosine to produce AV block.

Heart rate is important in determining the minimum effective dose of adenosine. The underlying ionic mechanisms have been studied using isolated AV nodal cells. Adenosine activated a potassium conductance $\left(\mathrm{I}_{\mathrm{KAdo}}\right)$ and reduced the inward calcium current $\left(\mathrm{I}_{\mathrm{ca}}\right){ }^{15}{ }^{16}$ decreasing the excitability of single AV nodal cells and enhancing rate dependent activation failure. ${ }^{16}$ In humans, the negative dromotropic effect induced by intravenous bolus injections of adenosine is more pronounced at fast atrial pacing rates. ${ }^{17}$ Lower doses of adenosine may therefore be effective at fast rates, for instance during supraventricular tachycardia. Over half of patients with supraventricular tachycardia respond to $6 \mathrm{mg}$ of adenosine, ${ }^{3}$ whereas in the present study the majority of patients in sinus rhythm required higher doses. This rate 
dependent effect of adenosine is of clinical benefit in allowing termination of tachycardia without subsequent $\mathrm{AV}$ block during restored sinus rhythm.

The reproducibility of repeated doses of adenosine was not tested in this study but has been reported previously. No significant intraindividual variations in the effects of five repeated doses of adenosine on $\mathrm{AV}$ conduction were observed during atrial pacing at constant rates. ${ }^{17}$ However, in the present study a dose which produced second degree AV block during the test injection failed to do so with the study dose in two of the patients, despite there being no significant difference in heart rates. This may have resulted from changes in autonomic tone following exercise testing, although studies have shown that neither muscarinic blockade with atropine nor catecholamine stimulation have significant effects on the actions of adenosine..$^{18}$

The site of action of adenosine at the AV node is long established but it has not been demonstrated whether this is from direct exposure of the nodal tissue to adenosine during its transit through the right atrium or from coronary perfusion of the node following passage of the bolus through the left heart. In isolated tissue preparations, superfusion with adenosine rendered nodal cells inexcitable and caused AV block. ${ }^{7}$ That the AV node is accessible from the right atrium in humans has been clearly demonstrated by recent experience with catheter ablation. ${ }^{20}$ However, the correlation of the electrophysiological effects of adenosine with the transit of the bolus in this study indicated that coronary perfusion was the more likely route of delivery.

Adenosine induced AV block was preceded by sinus slowing in about half the patients. Studies with isolated rabbit sino-atrial cells ${ }^{21}$ have shown that adenosine activated a potassium current, $\mathrm{I}_{\mathrm{KAdo}}$, as it does in $\mathrm{AV}$ nodal cells, ${ }^{15}$ although higher concentrations were required to cause complete arrest. In the present study, the earlier onset of sinus slowing suggested a greater sensitivity of the sinus node to adenosine compared with the $\mathrm{AV}$ node in some patients.

CONCLUSIONS

We showed marked differences between the electrophysiological effects of boluses of adenosine when given through a proximal arm vein compared with a distal hand vein. Imaging of the bolus showed that distal injections led to longer transit times and increased bolus dispersion, which provides an explanation for the reduced effect. In clinical practice it is important that adenosine is given rapidly through a large proximal peripheral vein, preferably in the antecubital fossa, followed by a saline flush to maximise efficacy. This may be of particular importance when adenosine is given as an aid to the diagnosis of broad complex tachycardia, when absence of effect may be taken as evidence that the arrhythmia is of ventricular origin. Our present results show that absence of effect may have as much to do with the method of administration as with the arrhythmia mechanism. Finally the correlation of the electrophysiological effects of adenosine with passage of the bolus through the left ventricle indicated the dependence of the negative chronotropic and dromotropic effects on coronary arterial delivery of adenosine to the nodal tissues.

1 Honey RM, Rithchie WT, Thomson WAR. The action of adenosine upon the human heart. Qf Med 1930;23:485-9. 2 DiMarco JP, Sellers TD, Berne RM, et al. Adenosine: electrophysiologic effects and therapeutic use for terminating paroxysmal supraventricular tachycardia. Circulation 1983; 68:1254-63.

3 Dimarco JP, Miles W, Akhtar M, et al, and the Adenosine for PSVT Study Group. Adenosine for paroxysmal supraventricular tachycardia: dose ranging and comparison with verapamil. Ann Intern Med 1990;113:104-10.

4 Rankin AC, Brooks R, Ruskin JN, et al. Adenosine and the treatment of supraventricular tachycardia. Am f Med 1992; 92:655-64.

5 Möser GH, Schrader J, Deussen A. Turnover of adenosine in plasma of human and dog blood. Am $\mathcal{F}$ Physiol 1989;256: C799-806.

6 Rankin AC, Oldroyd KG, Chong E, et al. Value and limitaions of adenosine in the diagnosis and treatment of narrow and broad complex tachycardias. Br Heart 7 1989;62:195203.

7 Clemo HF, Belardinelli L. Effect of adenosine on atrioventricular conduction. I. Site and characterization of adenosine action in the guinea pig atrioventricular node. Circ Res 1986;59:427-36.

8 Garratt CJ, Antoniou A, Griffith MJ, et al. Use of ntravenous adenosine in sinus rhythm as a diagnostic test for latent preexcitation. Am f Cardiol 1990;65:868-73.

9 Cohen TJ, Tucker KJ, Abbott JA, et al. Usefulness of adenosine in augmenting ventricular preexcitation for noninvasive localization of accessory pathways. Am F Cardiol 1992; 69:1178-85.

10 Keim S, Curtis AB, Belardinelli L, et al. Adenosine-induced atrioventricular block: a rapid and reliable method to assess surgical and radiofrequency catheter ablation of accessory atrioventricular pathways. f Am Coll Cardiol 1992;19:100512.

11 Walker KW, Silka MJ, Haupt D, et al. Use of adenosine to identify patients at risk for recurrence of accessory pathway conduction after initially successful radiofrequency catheter ablation. PACE 1995;18:441-6.

12 McIntosh-Yellin NL, Drew BJ, Scheinman MM. Safety and efficacy of central intravenous bolus administration of adenosine for termination of supraventricular tachycardia. f Am Coll Cardiol 1993;22:741-5.

13 Mubagwa K, Mullane K, Flameng W. Role of adenosine in the heart and circulation. Cardiovasc Res 1996;32:797-813.

14 Lai W-T, Lee C-S, Wu J-C, et al. Effects of verapamil, propranolol, and procainamide on adenosine-induced negative dromotropism in human beings. Am Heart f 1996;132: 768-75.

15 Martynyuk AE, Kane KA, Cobbe SM, et al. Adenosine increases potassium conductance in isolated rabbit atriov-
entricular nodal myocytes. Cardiovasc Res 1995;30:668-75.

16 Wang D, Shryock JC, Belardinelli L. Cellular basis for the negative dromotropic effect of adenosine on rabbit single atrioventricular nodal cells. Circ Res 1996;78:697-706.

17 Lai W-T, Lee C-S, Wu S-N. Rate-dependent properties of adenosine-induced negative dromotropism in humans. Circulation 1994;90:1832-9.

18 Favale S, DiBiase M, Rizzo U, et al. Effect of adenosine and adenosine-5'-triphosphate on atrioventricular conduction in patients. F Am Coll Cardiol 1985;5:1212-19.

19 Lauer MR, Young C, Liem B, et al. Efficacy of adenosine in terminating catecholamine-dependent supraventricular tachycardia. Am f Cardiol 1994;73:38-42.

20 Yeung-Lai-Wah JA, Alison JF, Lonergan L, et al. High success rate of atrioventricular node ablation with radiofrequency energy. F Am Coll Cardiol 1991;18:1753-8.

21 Belardinelli L, Giles WR, West A. Ionic mechanisms of adenosine actions in pacemaker cells from rabbit heart. $\mathcal{f}$ Physiol (Lond) 1988;405:615-33. 